\title{
Meta-Analysis of Randomized Controlled Trial in Treating Primary Liver Cancer by Fufang Kushen Injection Combined with TACE
}

\author{
Dehui Li ${ }^{1}$, Huanfang Fan ${ }^{1}$, Chunxia Sun ${ }^{1}$ \\ ${ }^{1}$ Chinese traditional medicine Hospital of Hebei province, Shijiazhuang, China, 050011 \\ alidehui2008@qq.com
}

\begin{abstract}
Keywords: Fufang Kushen Injection, TACE, Primary Liver Cancer, Meta analysis
\end{abstract}
Abstract. Objective. To evaluate effectiveness and safety of Fufang Kushen Injection combined with transcatheter arterial chemoembolization (TACE) for primary liver cancer (PLC). Search Strategy. Electronic databases were searched until 2 July, 2015. Inclusion Criteria. We included randomized clinical trials about comparison between Fufang Kushen Injection combined with TACE (FFKS-TACE) and TACE alone for treatment of PLC. Data Extraction and Analyses. Study selection, data extraction, quality assessment, and data analyses were conducted according to Cochrane standards. Results. 19 randomized trials were included. Methodological quality of the included trials was evaluated as generally low. compared with TACE alone, Meta-analysis showed that FFKS-TACE significantly improved the effective rate, KPS, six-month, one-year and two-year survival; significantly reduced the incidence of nausea and vomiting, fever, myelosuppression, transminase increased, bilirubin increased and stomach ache. Conclusion. FFKS-TACE for PLC can not only improve the effectiveness rate, KPS and survival rate, but also reduce the incidence rate of the clinical adverse effects such as nausea and vomiting, fever, myelosuppression, hepatic injury and stomach ache. However, the conclusion needs to be proved by further experiments on account of the absence of high quality articles. But evidence remains weak, rigorously designed trials are warranted to confirm these results.

\section{Introduction}

Primary Liver Cancer is one of the commonest forms of malignancies and the top three causes of cancer death [1]. In recent years, transarterial chemoembolization (TACE) is one of the preferred treatments for patients with PLC who are not suitable for surgery or postoperative recurrence. FFKS was extracted from the mixture of Sophora flavescens and Heterosmilacis Japonicae with the mass ratio of 7 than 3, Experimental studies showed that FFKS could inhibit tumor cell growth, alleviate pain, elevated white blood cell, anti-infection and protect the body immune function [2,3].Until now, Many clinical trials showed that FFKS-TACE can reduce the adverse reactions and improve quality of life for primary liver cancer (PLC). however, a multi- center, randomized, double-blind PlaceboControlled Trial of FFKS-TACE for Treatment of PLC have not been reported. In this paper to evaluate effectiveness and safety of FFKS-TACE for primary liver cancer (PLC) by meta- analysis, and provide evidence-based information for clinical practice.

\section{Research Data and Search Methods}

Source of Data. Literature searching is electronic retrieval, database of which contain CNKI, CSJD, CBM, Pubmed, Web of Science and Springer. The keywords are TACE, transcatheter arterial chemoembolization, Hepatocarcinoma, Hepatoma, liver cancer, compound Kushen Injection, Compound matrine injection. Retrieval date is until 2 July, 2015.

Inclusion Criteria.(1)Study types: The literature must be the randomized controlled trial (RCT). (2) primary liver cancer patients were confirmed cytologically or pathologically. (3)published trials included a treatment group receiving FFKS-TACE and a control group receiving TACE.(4) The study have not used other Chinese medicine anticancer drugs before it included. 
Exclusion Criteria. (1)Experiments design of literature was inexact, such as the criteria for the diagnosis and curative effect was non-standard; sample data did not make clear; evaluating indicator was ambiguous; etc. (2) statistical method was inappropriate. (3) Not therapeutic clinical research, animal studies or in vitro studies.

Quality Evaluation and Data Extraction. Each literature which fit the inclusion criteria was made quality evaluation and data extraction independently by two researchers in the unified quality evaluated form. The quality of literature which was included on the methodology used Jadad scale[4]. RCTs is 1-5 points. Literatures that were analyzed the Jadad scroe of which is 1or higher.

Outcome Indicators. main indicators: efficient (Response Evaluation Criteria In Solid Tumors, The overall response rate $\mathrm{CR}+\mathrm{PR}$ ), Survival rate, Karnofsky score (KPS). Secondary indicators: Adverse reactions, such as incidence of nausea, vomiting, myelosuppression, stomach ache, transminase and bilirubin increased.

Statistical Methods. The data are processed by RevMan4.2 software supported by the Cochrane Collaboration. Test of publishing bias are analyzed by funnel plot.

\section{Results}

Characteristics of Included Studies and Quality of the Methodology. 75 Related Articles were achieved By computer search, After reading the title, abstract and full text later, Eliminate unqualified 56 literature articles, Eligible included 19 literature, A total of 1370 cases. FFKS-TACE (692) and TACE (678).

Effective Rate. 17 literatures of Included studies were identified with a CR+PR outcome measurement of tumor response. The fixed-effects model was used because of heterogeneity of the results of trials (Heterogeneity: Chi2 $\left.=11.32, \mathrm{df}=16(P=0.0 .79) ; \mathrm{I}^{2}=0 \%\right)$. The pooled analysis showed that compared with TACE alone, FFKS-TACE significantly improved the effective rate $(\mathrm{OR}=1.99$; 95\% CI [1.55, 2.57]; $P<0.0001$ ) (refer with: Fig.1).

Quality of life. There were 9 trials contained a KPS improvement of $>10$ points. The fixed-effects model was used (Heterogeneity: Chi2 $\left.=5.68, \mathrm{df}=8(P=0.68) ; \mathrm{I}^{2}=0 \%\right)$. The results showed that FFKS+TACE significantly improved KPS $(\mathrm{OR}=2.81$; 95\% CI $[1.98,3.97]$; $P<0.00001)$ (refer with: Fig.2).

Survival Rates. 3 trials were identified with the outcome measurements of three-month survival. The fixed-effects model was used (Heterogeneity: Chi2 $\left.=0.05, \mathrm{df}=1 \quad(P=0.81) ; \mathrm{I}^{2}=0 \%\right)$. The meta-analysis showed that There was no statistically significant difference between FFKS+TACE and TACE alone. There are 5, 6 and 3 trials were identified with the outcome measurements of six-month, one-year and two-year survival respectively, The fixed- effects model was used $(P=0.98, P=0.90$ and $P=0.64$ respectively), six-month $(\mathrm{OR}=2.27,95 \% \mathrm{CI}[1.40,3.69], P=0.0009)$, one-year $(\mathrm{OR}=2.21$, 95\% CI $[1.45,3.36], P=0.0002)$ and two-year survival $(\mathrm{OR}=2.28,95 \% \mathrm{CI}[1.09,4.78], P=0.03)$. The meta-analysis showed that FFKS-TACE significantly improved the survival rate, the difference was statistically significant $(P=0.0009,0.0002$ and 0.03 respectively). 2 trials were identified with the outcome measurements of three-year survival. The fixed-effects model was used (Heterogeneity: Chi2=0.05, $\left.\mathrm{df}=1 \quad(P=0.81) ; \mathrm{I}^{2}=0 \%\right)$. The meta-analysis showed that There was no statistically significant difference between FFKS+TACE and TACE alone ( $P=0.07)$. (refer with: Fig.3).

Adverse reactions. There are 7 studies reported the incidence of nausea and vomiting, 6 for fever, 4 for myelosuppression, 7 for transminase increased, 4 for bilirubin increased and 4 for stomach ache. Studies $(P>0.05)$, the fixed-effects model was used, The meta-analysis showed that compared with TACE alone, FFKS-TACE significantly reduced the incidence of nausea and vomiting $(\mathrm{OR}=0.35$, 95\% CI[0.27, 0.58], $P<0.00001)$, fever(OR=0.26, 95\% CI [0.18, 0.40], $P<0.00001)$, myelosuppression $(\mathrm{OR}=0.36,95 \% \mathrm{CI}[0.22,0.59], P<0.00001)$, transminase increased $(\mathrm{OR}=0.28,95 \% \mathrm{CI}[0.19,0.43]$, $P<0.00001)$, bilirubin increased $(\mathrm{OR}=0.35,95 \% \mathrm{CI}[0.21,0.57], P<0.00001)$ and stomach ache $(\mathrm{OR}=0.31,95 \% \mathrm{CI}[0.19,0.50], P<0.00001)$. (refer with: Fig.4). 


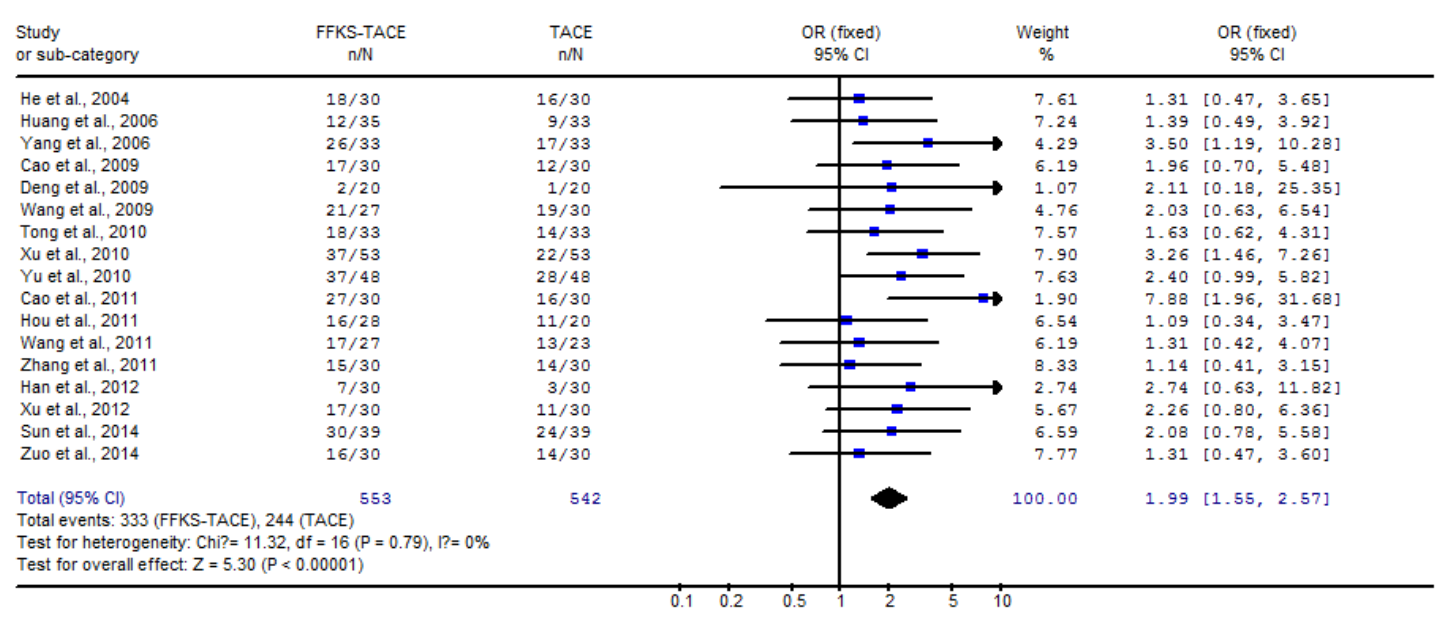

Figure 1 Meta-analysis of FFKS + TACE and TACE effective rate

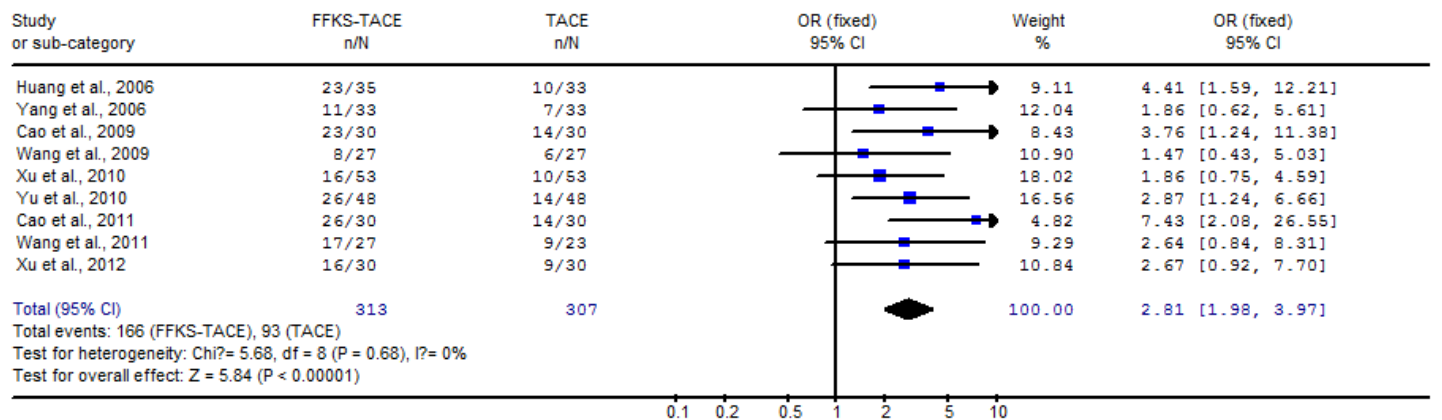

Figure 2 Meta-analysis of quality of life of FFKS+TACE and TACE

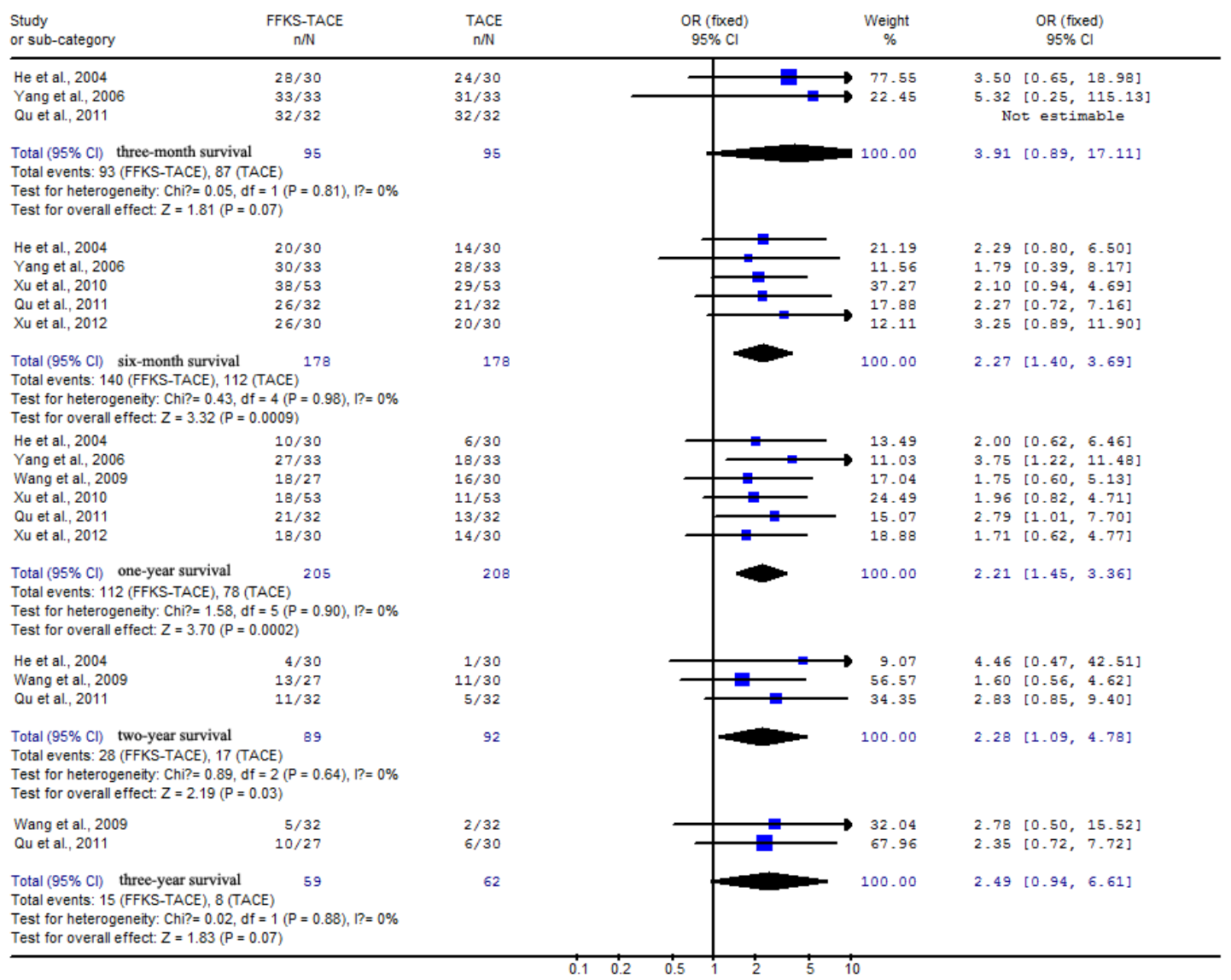

Figure 3 Meta-analysis of survival rates of FFKS+TACE and TACE 


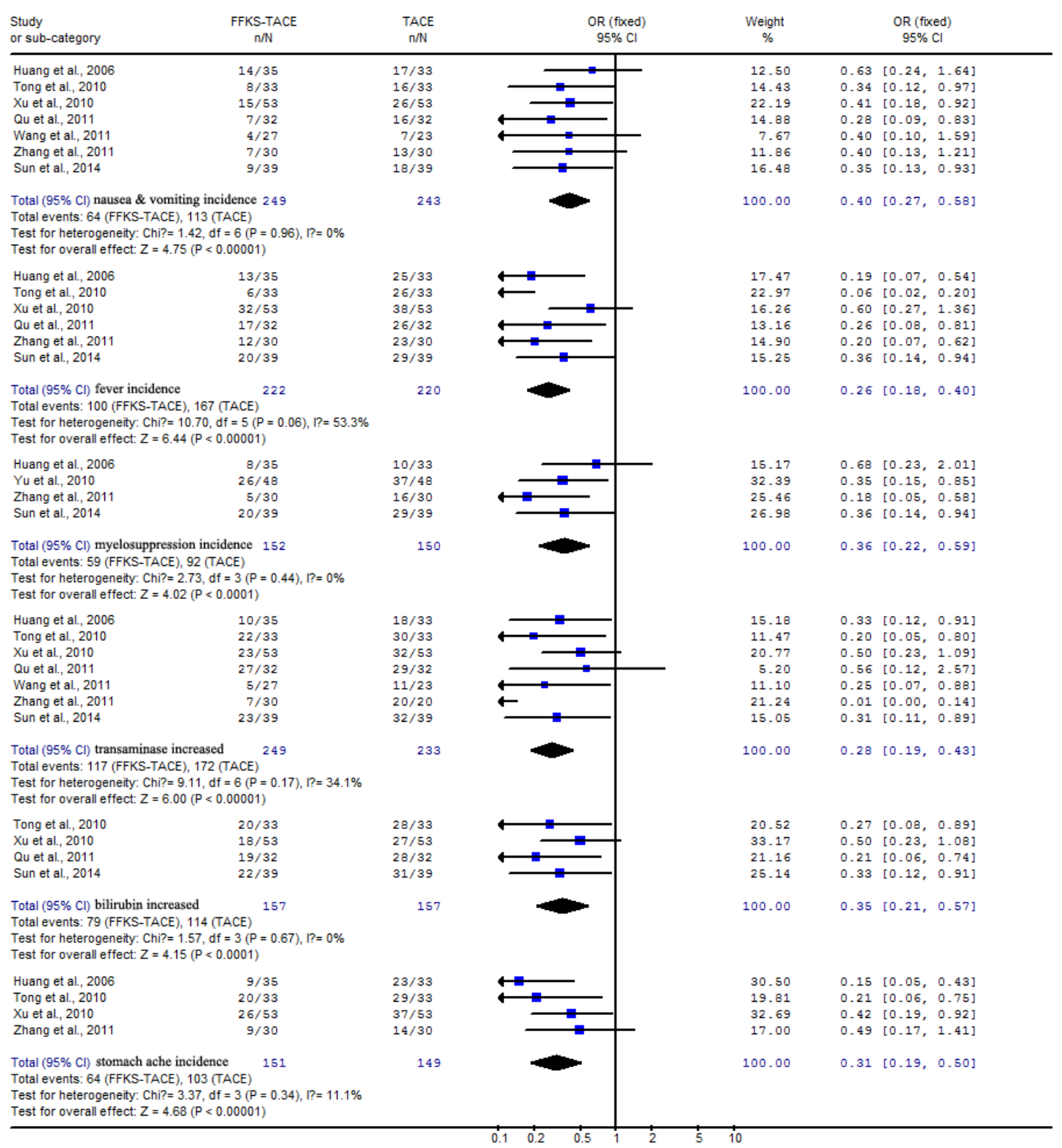

Figure 4 Meta-analysis of adverse reactions of FFKS+TACE and TACE

Publication Bias. funnel plot were performed to examine the publication bias of literatures (refer with: Fig.5). The shape of the funnel plots seemed symmetric, and did not reveal any evidence of publication obvious.

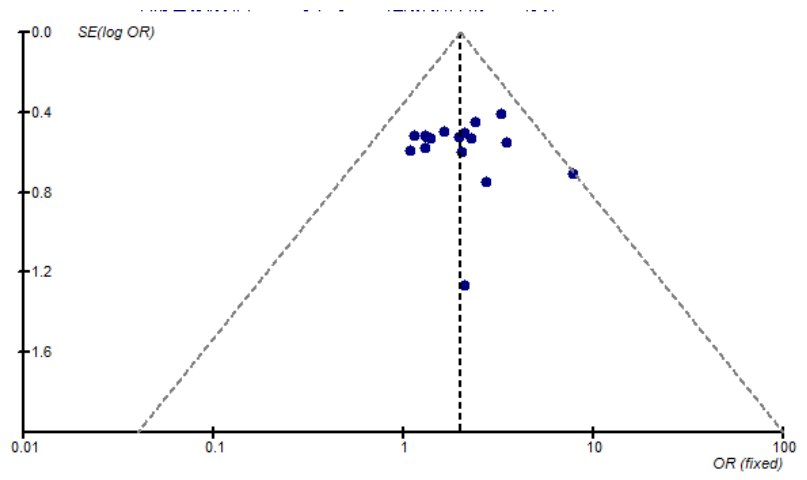

Figure 5 Funnel plot of effective rate (CR+PR). 


\section{Discussion}

Fufangkushen injection is a pure Chinese medicine, which consisted of the Sophora, Tuckahoe, indica, Wulingzhi, Polygonum and other Chinese herbal medicines. Experimental studies showed that FFKS could inhibit tumor cell growth, alleviate pain, stops bleeding, elevated white blood cell, anti-infection and protect the body immune function. Many clinical trials showed that Fufang Kushen Injection combined with TACE (FFKS-TACE) can reduce the adverse reactions and improve quality of life for PLC. However, a multi-center, randomized, double- blind, Placebo-Controlled trial of FFKS-TACE for Treatment of PLC have not been reported, so The effect of FFKS-TACE on the treatment of Primary Liver Cancer has not been recognized. This study Collect existing randomized clinical trials literature, use Evidence Based Medicine method, to evaluate effectiveness and safety of FFKS-TACE for PLC . And provide evidence-based information for clinical practice.

The results show that FFKS-TACE seemed superior to TACE alone for the treatment of primary liver cancer. FFKS-TACE for PLC can not only improve the effectiveness rate, KPS and survival rates, but also reduce the incidence rate of the clinical adverse effects such as nausea and vomiting, fever, myelosuppression, hepatic injury and stomach ache. However this system evaluation found the following problems that may affect the reliability of these conclusions. First, the quality of included literature are low, According to the Jadad scale, all the trials receive a Jadad score of 1-2. These literature rarely described the randomisation, double-blinding, dropouts and withdrawals, statistical methods and some literature baseline information in detailed, it may lead to selection bias when grouping of study, Performance bias when treating, expectation bias when therapeutic effect evaluation. Second, From the interventions of included study to see, the Chemotherapy and treatment cycle, drug dose and time of each study are not exactly the same, these all have a greater impact on the results of the evaluation. So it is worthy of additional study. Hence, larger, longer-term, rigorously designed, multi-center, randomized, double- blind, controlled trials are required to fully assess whether FFKS-TACE is more outstanding than TACE alone.

\section{Acknowledgements}

This work was financially supported by The Fundamental Research Funds for the Central Universities(No.216113119); Research projects of Traditional Chinese Medicine Administration of Hebei Province(No.2015104).

\section{References}

[1]. Y. Zhang, J.S. Ren, J.F. Shi, et al. International trends in primary liver cancer incidence from 1973 to 2007. BMC Cancer, vol. 15(2015), p. 94.

[2]. B. Yanju, L. Yang, B. Hua, et al. A systematic review and meta-analysis on the use of traditional Chinese medicine compound kushen injection for bone cancer pain. Support Care Cancer, vol. 22(2014), p. 825-836.

[3]. W. Wang, R.L. You, W.J. Qin, et al. Anti-tumor activities of active ingredients in Compound Kushen Injection. Acta Pharmacol Sin, vol. 36(2015), p. 676-679.

[4]. A.R. Jadad, R.A. Moore, D. Carroll, et al., Assessing the quality of reports of randomized clinical trials: is blinding necessary? Control Clin Trials, vol. 17(1996), p. 1-12. 